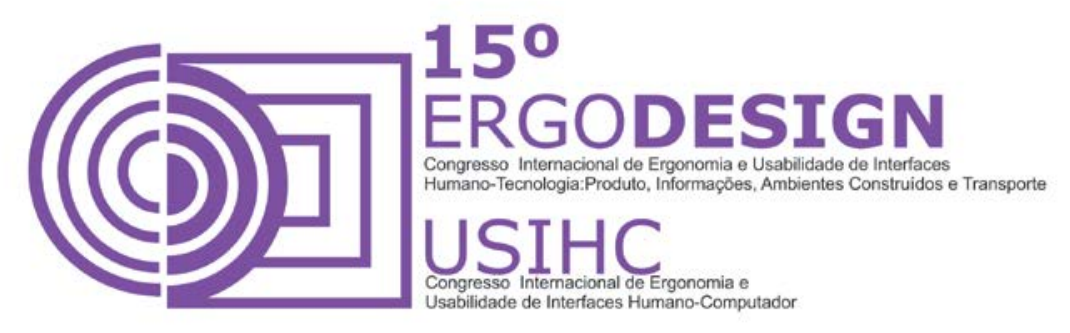

\title{
REDESENHO DA ÁREA DOS ALUNOS DO AMBIENTE VIRTUAL DE APRENDIZAGEM ADOTADO POR UMA UNIVERSIDADE FEDERAL
}

\section{REDESIGN OF THE STUDENTS' AREA ON A LEARNING OF VIRTUAL ENVIRONMENT ADOPTED BY A FEDERAL UNIVERSITY}

\author{
SILVA, Wanderson Rodrigues da ${ }^{(1)}$; \\ GABRIEL, Luiz Henrique de Lima ${ }^{(1)}$; \\ SILVA, Wélita Borges da ${ }^{(1)}$; \\ BOTELHO, Thais ${ }^{(1)}$; \\ PRIETCH, Soraia Silva ${ }^{(2)}$ \\ (1) Universidade Federal de Mato Grosso/ Rondonópolis, Graduando(a) \\ e-mail: wandersons.roo@hotmail.com, l.rickgabriel@hotmail.com, \\ welithaborges@yahoo.com.br, thaisbotelho13@gmail.com \\ (2) Universidade Federal de Mato Grosso/ Rondonópolis, Doutora \\ e-mail: soraia@ufmt.br
}

\begin{abstract}
RESUMO
Os cursos de graduação e pós-graduação de uma Universidade vêm utilizando um Ambiente Virtual de Aprendizagem (AVA) para complementação das atividades presenciais. No entanto, percebeu-se baixo acesso extraclasse pelos alunos, e acredita-se que problemas de usabilidade estejam associados a isto. Sendo assim, o objetivo deste trabalho é propor o redesenho da área dos alunos do AVA. Para isso um ciclo de três etapas foi adotado: análise contextual, concepção e avaliação. A análise dos resultados revelou que o redesenho do AVA aluno foi satisfatório, sendo que, de 120 possibilidades, 97 foram favoráveis durante a avaliação por participantes.
\end{abstract}

Palavras-chave: Interação Humano-Computador, Usabilidade, AVA, Avaliação com usuários.

\section{ABSTRACT}

A Virtual Learning Environment (VLE) has been used in undergraduate and graduate courses of an University, in order to supplement the classroom activities. However, it was noticed low access of the system by students, and we believe that usability problems are associated with this fact. Thus, the goal of this work is to propose a redesign of the students' area of the VLE. For this a three-step cycle was 


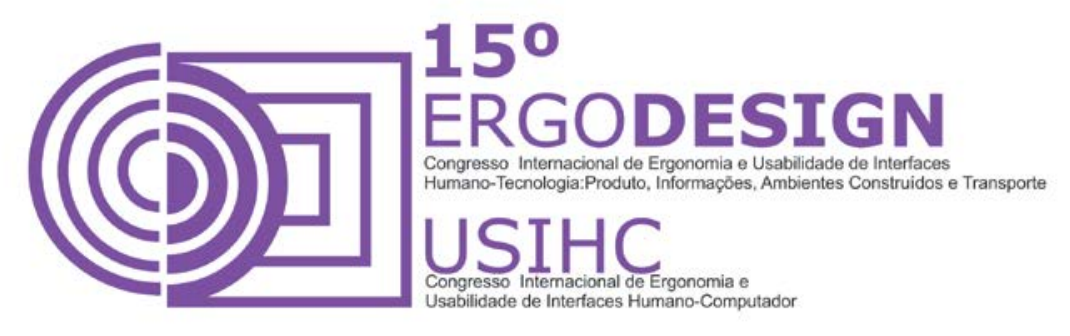

adopted so that this goal was achieved: contextual analysis, conception/prototyping, and evaluation. The results revealed that the redesign of the AVA was satisfactory; and, among 120 possibilities, 97 were considered favorable during the evaluation by participants.

Keywords: Human-Computer Interaction, Usability, LVE, users evaluation.

\section{INTRODUÇÃO}

Um dos desafios da comunidade de Interface Humano-Computador (IHC) é permitir que a evolução tecnológica ocorra de maneira adaptável para qualquer individuo que necessita ou deseja usufruir de uma determinada tecnologia. Projetos que não atendam princípios básicos de usabilidade podem acarretar confusão na interpretação do usuário final ao utilizar a tecnologia em mãos.

No que diz respeito à plataforma Moodle, que é um ambiente de vários recursos (tais como: chat, mensagens, fóruns, dentre diversas outras possibilidades), é frequente o não uso dos recursos pelos alunos, da Universidade Federal tratada neste artigo. Um ponto a ser destacado é que, a falta de usabilidade de um AVA pode impactar na falta de uso do mesmo, conforme estudo publicado por Magalhães et al (2010). Ainda, segundo Andrade e Neves (2012, p. 2), "a usabilidade do AVA não deve atrapalhar a motivação de um aluno, pois ao utilizar um sistema agradável, ele se compromete e obtém sucesso em um curso". Além disso, pressupõe que os alunos só acessem o AVA a pedido do professor, obtendo uma experiência de uso negativa, por ser obrigatória e não voluntária (VENKATESH et al, 2003).

Diante da problemática apresentada, um grupo de estudantes da disciplina Interface HomemMáquina do curso de Sistemas de Informação de uma Universidade Federal, resolveu conciliar o aprendizado da disciplina com o AVA adotado pela Universidade. No decorrer da disciplina, cada novo conteúdo teórico aprendido, era empregado na prática do ciclo de IHC.

O objetivo, então, do presente projeto era o de realizar uma proposta de redesenho do AVA aluno, a fim de verificar se o baixo acesso ao ambiente tem relação com falhas de usabilidade. Para atingir o objetivo, foi definido um ciclo de três etapas, as quais são descritas na Seção 2.

\section{METODOLOGIA DE PESQUISA}

O presente projeto foi proposto na disciplina Interface Homem-Máquina de um curso de Sistemas de Informação, para o qual se definiu um ciclo de IHC em três etapas: análise contextual, concepção de protótipo, e avaliação do protótipo junto ao público-alvo.

A primeira etapa, de análise contextual, contemplou as seguintes ações: (a) O checklist da ErgoList $^{1}$ é composto por 18 critérios, sendo que cada critério possui um rol de questões específicas (Tabela 2). Todos os critérios foram avaliados, e como resultado foi disponibilizado um laudo final com o número de questões respondidas e as observações feitas em algumas

\footnotetext{
${ }^{1}$ Disponível em: http://www.labiutil.inf.ufsc.br/ergolist/.
} 


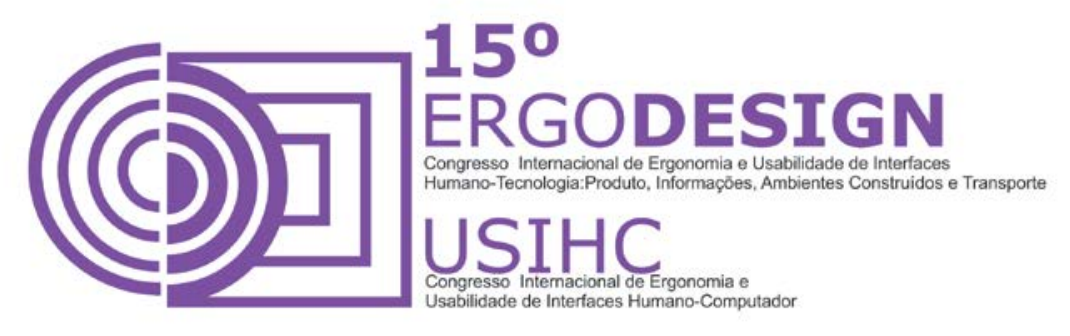

das questões; (b) Para análise de acessibilidade, o avaliador automático A-Checker ${ }^{2}$ foi utilizado. Ele fornece um relatório de todos os erros encontrados, considerando as normas da WCAG 2.03; e (c) O uso da técnica de brainstorming (MELLO e BARANAUSKAS, 2005) foi importante para elencar novas ideias não evidenciadas somente pela correção de falhas de usabilidade e/ou de acessibilidade. A elucidação dos requisitos funcionais e não funcionais, portanto, levaram em consideração o resultado da inspeção com o ErgoList, da avaliação automática de acessibilidade, da revisão de literatura, e do resultado do brainstorming.

A segunda etapa, de concepção do protótipo, levou em consideração as seguintes ações: (a) A técnica de card sorting (CYBIS, BETIOL e FAUST (2007); (CAMARGO e VIDOTTI (2011)) foi feita na modalidade aberta para a verificação do mapa mental da equipe; (b) Foi utilizado o bookmarklet do Wirify ${ }^{4}$ para gerar automaticamente os wireframe (GREENBERG et al (2012); MELO e BARANAUSKAS (2005)) atuais das telas: Home, Perfil e Disciplinas; sendo que seus itens foram descritos manualmente. Vários wireframe de propostas de redesenho foram esboçadas em papel e com o software Balsamiq ${ }^{5}$; (c) Alguns storyboard (GREENBERG et al (2012); MELO e BARANAUSKAS (2005)) foram produzidos utilizando a plataforma Storyboard That $^{6}$, de modo a prever alguns cenários de contexto de uso; e, (d) A concepção do protótipo de baixa fidelidade ocorreu em três passos, os quais são descritos na sequência: (i) Busca de ícones representativos e ideias de interface em sistemas correlatos, utilizados no protótipo; (ii) Organização dos ícones na interface, usando o Photoshop CS6; e, (iii) Concepção do protótipo final. A ferramenta Power Point 2013 foi utilizada para adicionar os hiperlinks às interfaces concebidas no passo dois, de modo a tornar o protótipo navegável.

A terceira etapa, de avaliação do protótipo junto ao usuário final, iniciou com a elaboração dos seguintes documentos: roteiro de avaliação, carta-convite ao usuário, termo de consentimento livre e esclarecido, lista de tarefas, ficha de observação, questionário de perfil e questionário de avaliação final. Vale mencionar que o questionário de avaliação foi elaborado com base em algumas questões da Ergolist, incluindo também questões sobre atributos da qualidade hedônica, de recomendação, experiência de uso, e sobre avaliação dos requisitos presentes; gerando, no total, 15 questões de avaliação.

\section{EMBASAMENTO TEÓRICO}

Além dos referenciais teóricos da área de IHC utilizados ao longo do desenvolvimento do ciclo, formado por projeto, concepção e avaliação, já citados nas seções anteriores; ainda realizou-se uma busca exploratória com o intuito de averiguar a existência de trabalhos correlatos. Com isso, verificou-se diversos relatos de investigações relacionadas ao tema de interesse do presente trabalho, realizadas em diferentes ambientes virtuais de aprendizagem de diversas

\footnotetext{
${ }^{2}$ Disponível em: http://achecker.ca/checker/index.php.

${ }^{3}$ Disponível em: http://www.w3.org/TR/WCAG20/.

${ }^{4}$ Disponível em: http://www.wirify.com/.

${ }^{5}$ Disponível em: https://balsamiq.com/.

${ }^{6}$ Disponível em: http://www.storyboardthat.com/.
} 


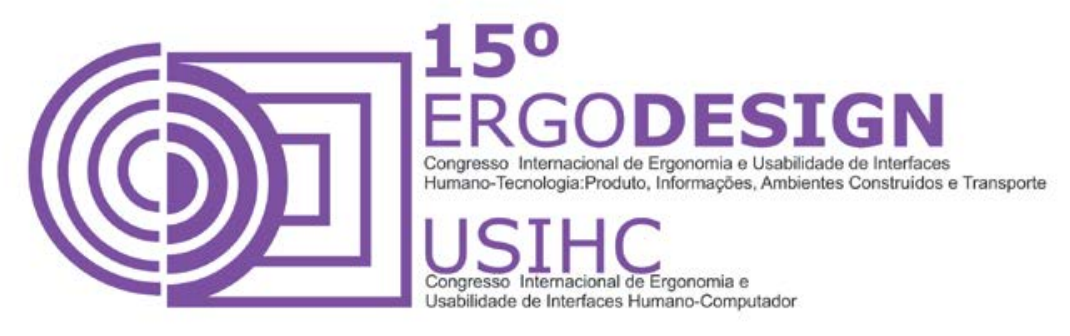

instituições de ensino superior brasileiras. Isso significa que a preocupação apontada anteriormente, a respeito do impacto de aspectos de usabilidade no uso de AVA é um tema relevante e recorrente. A Tabela 1 apresenta alguns dos trabalhos correlatos localizados, bem como os tipos de avaliação realizados em AVA abordados pelos pesquisadores.

Tabela 1 - Tipos de avaliação realizados em ambientes virtuais de aprendizagem.

\begin{tabular}{|l|l|}
\hline \multicolumn{1}{|c|}{ Tipo de avaliação } & \multicolumn{1}{c|}{ Referências } \\
\hline Análise de acessibilidade & Silveira e Batista (2011); Silva et al (2013) \\
\hline Teste de usabilidade com alunos & Carvalho e Eliasquevici (2013) \\
\hline $\begin{array}{l}\text { Comparativo de aspectos de usabilidade } \\
\text { concernente a diferentes AVA }\end{array}$ & Rocha e Palazon (2012); Carvalho et al (2014) \\
\hline Avaliação de usabilidade e navegabilidade & Barbosa et al (2012) \\
\hline
\end{tabular}

Considera-se importante mencionar que o presente trabalho agrega avalições a respeito da usabilidade, acessibilidade e arquitetura da informação; sendo realizadas inspeções técnicas, bem como avaliação junto ao público-alvo (alunos usuários do AVA em questão).

\section{DESENVOLVIMENTO DO CICLO BÁSICO DE IHC}

O ciclo de desenvolvimento envolveu as três etapas: análise contextual, concepção e avaliação do protótipo final. Dentre essas 3 etapas, somente na última houve participação do usuário final. Vale ressaltar que os integrantes da equipe (projetistas) também eram usuários do sistema.

\subsection{Análise contextual}

Do estudo da inspeção usando o checklist da ErgoList, obteve-se o resultado geral apresentado na Tabela 2, sendo que todas as questões foram respondidas.

Tabela 2 - Laudo final da inspeção com a ErgoList.

\begin{tabular}{|c|c|c|c|c|}
\hline Critérios avaliados & $\begin{array}{c}\text { Total de } \\
\text { questões }\end{array}$ & $\begin{array}{c}\text { Questões } \\
\text { conformes }\end{array}$ & $\begin{array}{c}\text { Questões não } \\
\text { conformes }\end{array}$ & $\begin{array}{c}\text { Questões não } \\
\text { aplicáveis }\end{array}$ \\
\hline 1. Concisão & 14 & 2 & 5 & 7 \\
\hline 2. Mensagens de erro & 9 & 5 & 3 & 1 \\
\hline 3. $\quad$ Flexibilidade & 3 & 0 & 1 & 2 \\
\hline 4. $\quad$ Legibilidade & 27 & 17 & 3 & 7 \\
\hline 5. Significados & 12 & 2 & 3 & 7 \\
\hline 6. Proteção contra erros & 7 & 0 & 2 & 5 \\
\hline 7. Agrupamento por formato & 17 & 10 & 0 & 7 \\
\hline 8. Experiência do usuário & 6 & 0 & 3 & 3 \\
\hline 9. Presteza & 17 & 6 & 3 & 8 \\
\hline 10. Controle do usuário & 4 & 0 & 0 & 4 \\
\hline 11. Correção de erros & 5 & 0 & 0 & 5 \\
\hline
\end{tabular}




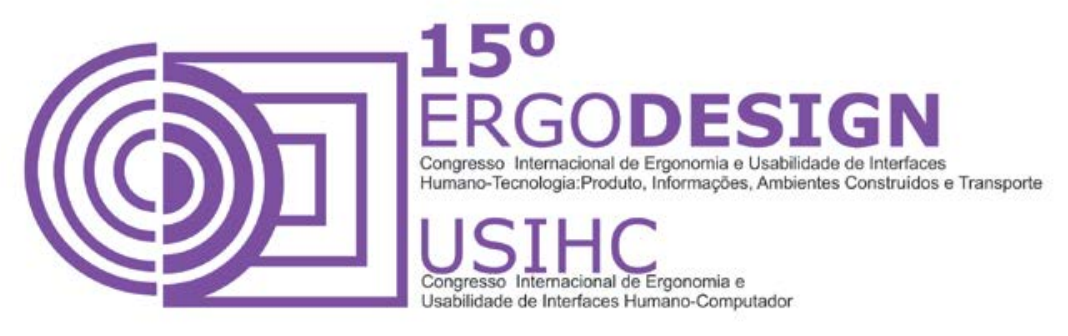

\begin{tabular}{|l|c|c|c|c|}
\hline Critérios avaliados & $\begin{array}{c}\text { Total de } \\
\text { questões }\end{array}$ & $\begin{array}{c}\text { Questões } \\
\text { conformes }\end{array}$ & $\begin{array}{c}\text { Questões não } \\
\text { conformes }\end{array}$ & $\begin{array}{c}\text { Questões não } \\
\text { aplicáveis }\end{array}$ \\
\hline 12. Consistência & 11 & 6 & 1 & 4 \\
\hline 13. Agrupamento por localização & 11 & 3 & 1 & 7 \\
\hline 14. Densidade informacional & 9 & 4 & 2 & 3 \\
\hline 15. Feedback & 12 & 2 & 8 & 2 \\
\hline 16. Compatibilidade & 21 & 8 & 1 & 12 \\
\hline 17. Ações explícitas & 4 & 2 & 0 & 1 \\
\hline 18. Ações mínimas & 5 & 2 & 2 & 1 \\
\hline TOTAL & 194 & 69 & 38 & 86 \\
\hline
\end{tabular}

Vale ressaltar que as questões foram respondidas considerando todas as telas passíveis de acesso pelos alunos, totalizando em 19 telas. Sendo assim, o laudo resultante agrega a avaliação de diferentes telas. Pode-se observar na Tabela 2 que diversos itens são não aplicáveis, pois os alunos possuem acesso mais passivo no AVA do que a área reservada para os professores.

Ao comparar o quantitativo de itens em conforme com os itens em não conforme, é possível verificar que existem mais itens em conforme. No entanto, o resultado ainda é favorável para continuidade no projeto para a proposição de melhorias nas telas do ambiente.

No que tange ao critério significados, as denominações dos títulos não estão de acordo com o que eles representam e que os títulos das páginas de menu não são explicativos, refletindo a natureza da escolha a ser feita. Essas respostas não são coerentes com a $1^{\text {a }}$ Lei de Krug (KRUG, 2008), o qual afirma "não me faça pensar". Um exemplo disso consta na Figura 1 (a), visto que quando o hiperlink "Voltar ao portal" é clicado, ao invés de voltar à página do portal, aparece uma tela em branco. Do critério feedback, na primeira questão por exemplo, foi identificado que o ambiente não fornece feedback adequado ao usuário, como por exemplo acesso de hiperlinks.

(a)

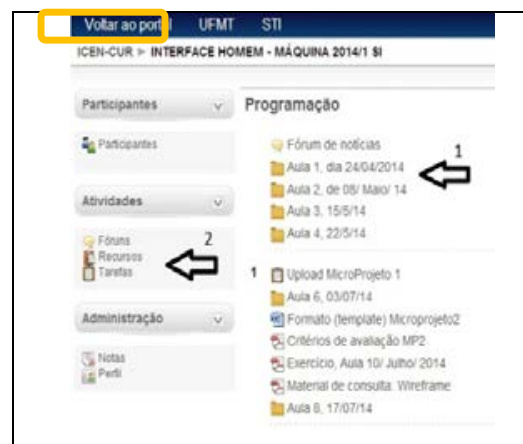

(b)

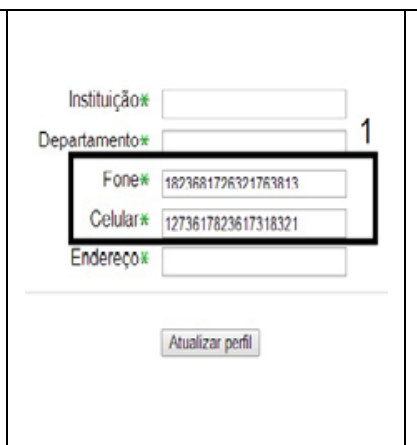

(c)

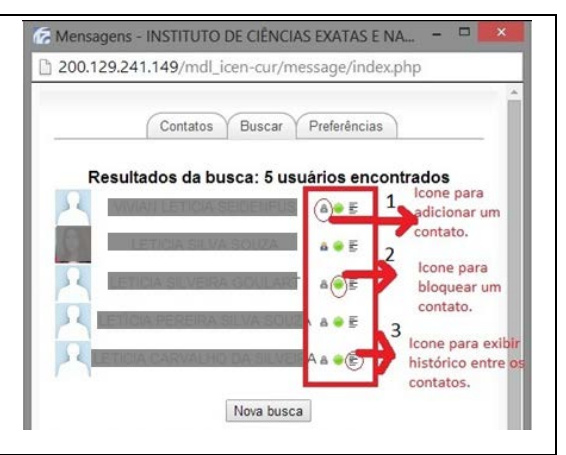

Figura 1. Exemplo de falha no critério: (a) feedback; (b) presteza; (c) legibilidade.

Na Figura 1 (a), apresenta a indicação para a resposta da pergunta do ErgoList: "o sistemas fornece feedback de todas as ações do usuário?"; para a qual a resposta é negativa, pois foi identificado que nesta tela que o usuário não sabe em qual pasta ou hiperlink ele já acessou 


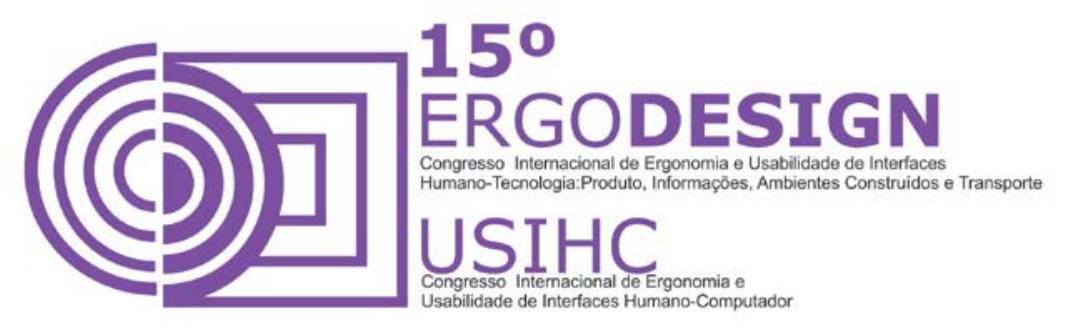

anteriormente. Os hiperlinks acessados continuam com a mesma cor que estavam antes do acesso.

No que se refere ao critério presteza, avaliando a tela de atualizar perfil, para a pergunta "Caso o dado a entrar possua um formato particular, esse formato encontra-se descrito na tela?", obteve-se uma negativa para os campos fone e celular; conforme pode ser visto na Figura 1 (b). Com relação ao critério de legibilidade, uma das questões é a seguinte: "Os ícones são legíveis?"; para qual também foi verificada uma negativa, pois foi identificado que nesta tela os ícones (destacados como 1, 2 e 3, na Figura 1 (c)) não são legíveis a todos os usuários, especialmente quando se deseja buscar outro usuário para trocar mensagens.

O uso de avaliadores automáticos de acessibilidade foi bastante útil, pois eles apontam diretamente onde há os problemas. Através do avaliador A-Checker, foi identificado, por exemplo, que o ambiente não fornece um texto explicativo para conteúdos não visuais e não há teclas de atalhos para acesso aos recursos. Além disso, a técnica de brainstorming realizada serviu também para descobertas de novos requisitos. A equipe se reuniu extraclasse para a realização desta atividade.

$\mathrm{Na}$ sequência seguem alguns exemplos de requisitos funcionais de usuário identificados: (a) Acessar o sistema de gerenciamento do acervo da biblioteca da Universidade, ou o sistema de gestão acadêmica da Universidade através do AVA; (b) Efetuar indicações materiais, em sugestões de endereços eletrônicos ou upload de arquivos; (c) Iniciar um novo tópico para discussão em Fórum ou Chat, por disciplina; (d) Poder configurar no AVA a recepção ou não por e-mail de aviso que o professor de determinada disciplina efetuou upload de novo material de aula no AVA. Ao total, foram 9 (nove) requisitos funcionais de usuário identificados através da técnica de brainstorming e da inspeção de sistemas correlatos.

A seguir constam alguns exemplos de requisitos não funcionais de usuário: (a) Escolher a opção de contraste de cores; (b) Efetuar a descrição das imagens do AVA para serem passíveis de reconhecimento por leitores de tela; (c) Possibilitar o redimensionamento, o corte, e a exclusão da imagem do perfil; (d) Após o envio de algum arquivo, apresentar uma mensagem informativa se houve sucesso no envio ou não; (e) Oferecer acesso opcional de um vídeo (tour) do AVA no primeiro acesso. Ao total, foram 10 (dez) requisitos não funcionais de usuário identificados através da técnica de brainstorming.

O público-alvo do projeto foi definido com base nos próprios integrantes da equipe e colegas de curso de graduação. O objetivo dessa definição foi para auxiliar o projeto centrado no usuário, pensando em suas necessidades específicas, bem como para determinar a participação de potenciais usuários na etapa 3 (avaliação). Neste sentido, pretendia-se que o protótipo fosse avaliado por pessoas matriculadas em cursos de graduação da Universidade.

\subsection{Concepção}

A técnica de card sorting auxiliou no redesenho do mapa do site, tomando como base o mapa mental da equipe do projeto e os princípios da arquitetura de informação. Além disso, estudar os wireframe gerados automaticamente, além de incluir as legendas explicativas, serviu para a 


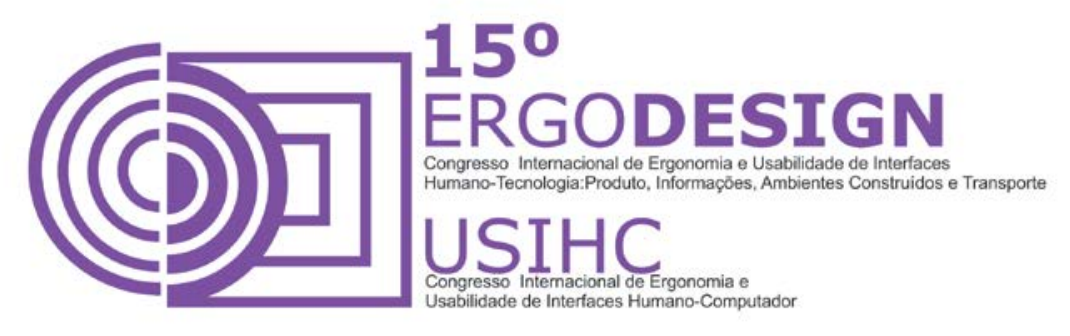

equipe conseguir evidenciar as principais diferenças entre os wireframe atuais (interface corrente) com os propostos, conforme mostra a Figura 2.

Para o desenho dos wireframe utilizou-se, em alguns momentos, um software e, em outros, papel e caneta. Com isso, foi possível verificar que esboçando em papel e caneta, tem-se uma maior liberdade de imaginação, além de poder rabiscar e tornar o processo de criação mais dinâmico entre a equipe. O software também tem a opção de desfazer, porém os recursos são mais limitados. Ficou evidente que o software fornece maior praticidade, além de deixar a interface mais organizada, e no papel, os esboços são mais rápidos.

(a)

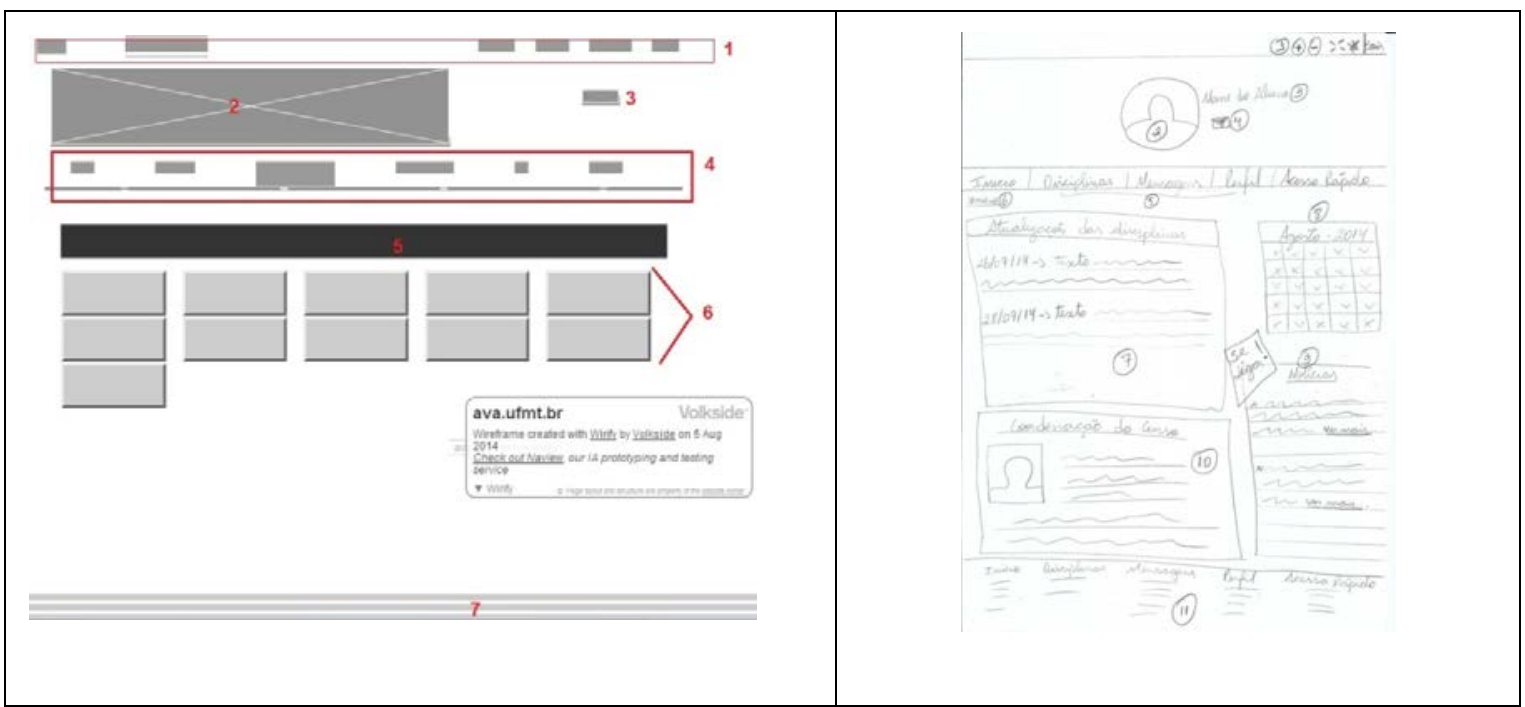

Figura 2. Exemplos dos wireframe da tela inicial (home): (a) atual; (b) proposto.

$\mathrm{O}$ acesso à página de disciplina que era feito clicando em algum ícone do campo 6 do wireframe atual (Figura 2(a)), agora o acesso passou a ser feito através do item de menu 'Disciplinas' do wireframe proposto (Figura 2(b)).

O botão 'Sair' do wireframe atual (item 3 da Figura 2(a)), que somente aparece na página 'Home', foi fixado no wireframe proposto no topo (item 1 da Figura 2(b)) para estar disponível em qualquer página do ambiente. Incluíram-se também os ícones para controle de contraste (entre as cores do plano de fundo e a cor da fonte) e para a alteração do tamanho da fonte.

No wireframe proposto (Figura 2(b)), o usuário pode acessar seu perfil clicando em seu nome ao lado da imagem, além de ter acesso às suas mensagens clicando no ícone de mensagens abaixo de seu nome; sendo que essas duas opções não são disponíveis no sistema atual. A imagem mudou de formato, de retangular para circular, e ficou centralizada no topo da página.

Foi incluído também, no wireframe proposto (Figura 2(b)), o campo de "atualizações das disciplinas", no qual os usuários podem ter acesso direto à página da disciplina clicando no 


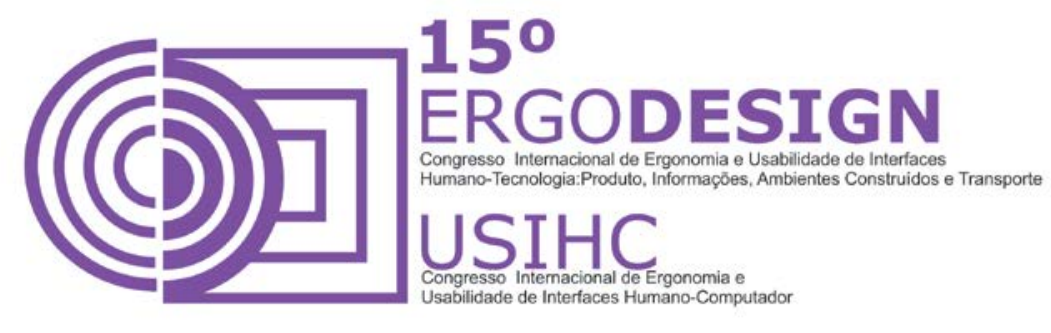

hiperlink da atualização. Além disso, alterou-se o "calendário" para um formato mais explicito, facilitando o acesso aos eventos e configurações.

Outra inclusão foi o campo de "noticiais", no qual a ideia é que sejam inseridas notícias relacionadas ao curso, ou à instituição; conferindo acesso direto à página dessas notícias através de hiperlinks. Outrossim, o mapa do site foi inserido na parte inferior para auxiliar a navegação do usuário pelo ambiente. Esses recursos não estavam disponíveis no wireframe atual (Figura 2(a)), exceto pelo calendário mostrando apenas a data atual.

A equipe também pode comparar as principais evidencias da página atual (interface corrente do AVA) com o protótipo. O protótipo criado foi de baixa fidelidade, desenvolvido com o Photoshop CS6, o qual incluiu as telas: Home, Perfil e Disciplinas, conforme mostra a Figura 3.

(a)

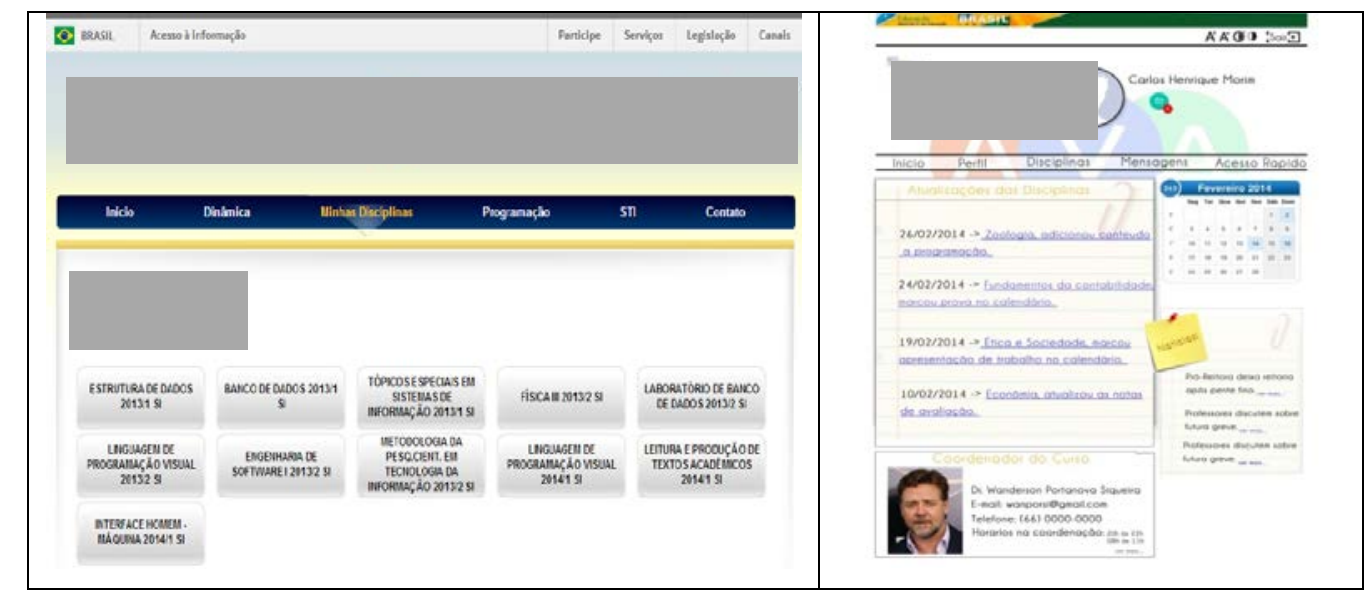

Figura 3. Exemplo das principais diferenças evidenciadas da tela inicial (home): (a) captura da tela atual do ambiente; (b) interface do protótipo.

Houve mudanças de toda a interface do ambiente, porém tentou preservar o esquema de cores que o ambiente já utilizava. Os botões que conferiam acesso às disciplinas, na Figura 3(a), foram retirados e acrescidos: (a) Atualizações das disciplinas: toda vez que um professor de uma determinada disciplina efetuar alteração na programação, este campo aparece em forma de hiperlink para que o usuário tenha acesso direto à informação; (b) Calendário: todos os eventos (global, pessoal, de disciplinas) agora estão visíveis na página inicial. Ao clicar no calendário, o usuário é direcionado à página de eventos, e pode a partir de então ter acesso aos agendamentos marcados; (c) Notícias: campo destinado às noticias gerais da Universidade; (d) Coordenador do curso: campo destinado ao coordenador do curso para disponibilizar informações pessoais e de contato; (e) Inclusão de ícones de acessibilidade; (f) Mapa do site no rodapé; (g) Mudança dos itens de menu: retirou-se o menu "Dinâmica", "Minhas disciplinas", "Programação", "STI" e "Contato"; e, foram incluídos: "Disciplinas" (com subitens de menu: disciplinas cursadas e em andamento), "Perfil", "Mensagens" e "Acesso rápido". 


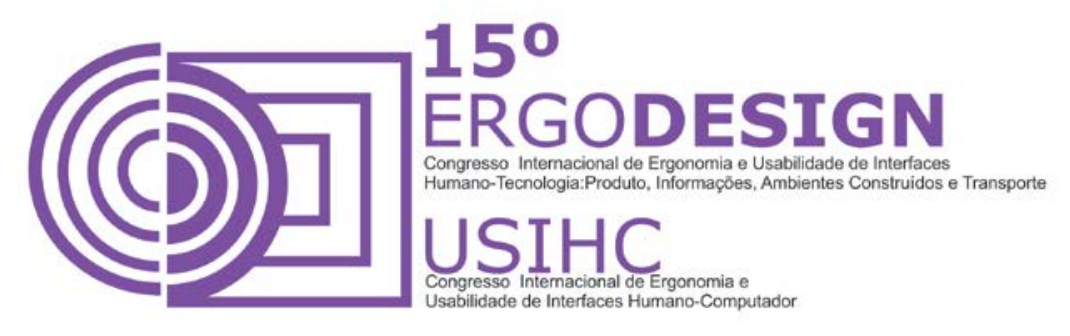

\subsection{Avaliação do protótipo (redesenho do AVA alunos)}

Para esta finalidade, foi realizado um teste piloto e um teste efetivo. Em ambos os testes, previamente foram estabelecidos dois critérios: (1) avaliação individual; e (2) o usuário deveria conhecer a plataforma AVA aluno. Ambos os testes tiveram a mesma sequência de ações realizadas, a saber: (a) Definição do local, datas e horários de realização; (b) Determinação dos equipamentos necessários e das funções dos membros da equipe durante a realização da avaliação; (c) Elaboração da documentação; (d) Convite e recrutamento dos participantes; (e) Leitura, explicação e assinatura do termo de consentimento; (f) Aplicação do questionário de perfil; (g) Realização das tarefas pelo usuário e anotação de comentários adicionais na ficha de observação pela equipe; e, (h) Aplicação do questionário de avaliação do protótipo.

O teste piloto foi realizado para identificar (caso houvesse) falhas no roteiro e na documentação elaborada pela equipe. Nesta fase, 05 (cinco) participantes (denominados aqui de P1 a P5), individualmente, desenvolveram as tarefas estabelecidas pela equipe, bem como responderam aos questionários de perfil e avaliação. O teste piloto contou com $100 \%$ dos participantes do curso de Sistemas de Informação, sendo estes veteranos; dentre os quais 04 são homens e 01 mulher, e $90 \%$ informou ter habilidade com informática em geral.

No questionário de avaliação, os usuários mencionaram algumas dificuldades para o entendimento de como marcar a opção de resposta desejada dentre as alternativas disponíveis. A equipe de projeto, então, efetuou as devidas adequações, de modo que durante o teste efetivo os participantes tivessem mais facilidade para compreender o questionário. Além disso, foi incluso um campo para que o usuário escrevesse sugestões, caso desejasse.

Após o teste piloto realizado, a avaliação efetiva foi concretizada. O intuito era avaliar se os erros apontados pela Ergolist foram sanados, bem como se o redesenho proposto atendeu ao público-alvo. $\mathrm{Na}$ avaliação efetiva, 08 (oito) estudantes de graduação participaram (denominados aqui de E1 a E8), sendo 1 do curdo de pedagogia, 2 de ciências contábeis, 3 de ciências econômicas, 1 de enfermagem e 1 de administração. Dentre os oito participantes, 5 eram do gênero masculino e 3 do gênero feminino; 6 eram veteranos e 2 calouros; e 07 participantes disseram ter habilidade com informática em geral. Em média, cada avaliação efetiva individual levou 20 minutos contemplando todas as atividades previstas no roteiro.

O questionário de avaliação, do teste efetivo, contou com 15 perguntas ou afirmações, as quais poderiam ser marcadas com opções fechadas de respostas, a saber: A = Concordo fortemente; $\mathrm{B}=$ Concordo; $\mathrm{C}=$ Nem concordo, nem discordo; $\mathrm{D}=$ Discordo; $\mathrm{E}=$ Discordo fortemente. As respostas dos participantes conferidas a este questionário constam descritas na Tabela 3.

Tabela 3 - Respostas ao questionário de avaliação (teste efetivo).

\begin{tabular}{|l|c|c|c|c|c|c|c|c|c|}
\hline Perguntas/ Afirmações & E1 & E2 & E3 & E4 & E5 & E6 & E7 & E8 & Percentual \\
\hline 1. Os ícones são claros/ representativos? & A & B & D & B & B & B & A & B & $70 \%$ B \\
\hline 2. Os ícones estão de tamanho adequado? & A & B & B & C & B & B & B & B & $80 \%$ B \\
\hline $\begin{array}{l}\text { 3. Não ficaram evidentes quais eram os campos } \\
\text { para edição do perfil? }\end{array}$ & C & E & D & A & D & D & C & D & $60 \% \mathrm{D}$ \\
\hline 4. Os títulos dos menus são explicativos, & A & B & A & B & B & A & A & B & $50 \%$ A, 50\% B \\
\hline
\end{tabular}




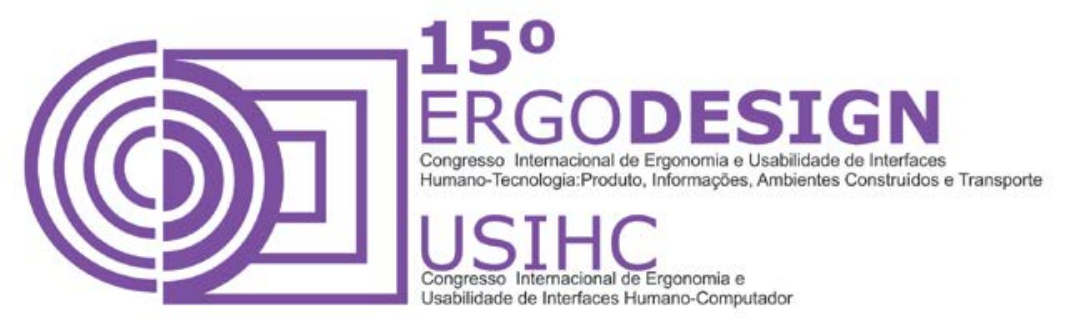

\begin{tabular}{|c|c|c|c|c|c|c|c|c|c|}
\hline \multirow{2}{*}{\multicolumn{10}{|c|}{$\begin{array}{l}\text { Perguntas/ Afirmações } \\
\text { refletindo a natureza da escolha a ser feita? }\end{array}$}} \\
\hline & & & & & & & & & \\
\hline 5. Os rótulos/ itens de menu são ambíguos. & $\mathrm{C}$ & $\mathrm{E}$ & $\mathrm{D}$ & $\mathrm{D}$ & $\mathrm{B}$ & $\mathrm{E}$ & $\mathrm{D}$ & $\mathrm{D}$ & $60 \% \mathrm{D}$ \\
\hline $\begin{array}{l}\text { 6. O AVA aluno proposto está mais intuitivo que } \\
\text { o atual? }\end{array}$ & A & B & C & A & B & A & $\mathrm{C}$ & B & $40 \%$ A, $40 \%$ B, $20 \%$ C \\
\hline $\begin{array}{l}\text { 7. Eu acessaria o AVA aluno para outros fins } \\
\text { senão ao comando do professor? }\end{array}$ & A & $\mathrm{D}$ & B & A & C & $\mathrm{C}$ & $\mathrm{C}$ & C & $\begin{array}{c}50 \% \mathrm{C}, 30 \% \mathrm{~A}, 10 \% \mathrm{D} \\
10 \% \mathrm{~B}\end{array}$ \\
\hline $\begin{array}{l}\text { 8. Não me senti confortável em utilizar o AVA } \\
\text { aluno sugerido. }\end{array}$ & $\mathrm{E}$ & $\mathrm{D}$ & $\mathrm{E}$ & D & D & $\mathrm{D}$ & $\mathrm{D}$ & $\mathrm{E}$ & $70 \% \mathrm{D}$ \\
\hline 9. Eu usaria o sistema frequentemente. & A & $\mathrm{B}$ & $\mathrm{B}$ & A & $\mathrm{B}$ & $\mathrm{B}$ & $\mathrm{B}$ & A & $70 \% \mathrm{~B}$ \\
\hline $\begin{array}{l}\text { 10. Eu considero que os alunos da } \\
\text { Universidade, independente do curso, } \\
\text { aprenderiam utilizar o sistema rapidamente? }\end{array}$ & A & B & B & B & B & $\mathrm{B}$ & A & B & $80 \% \mathrm{~B}$ \\
\hline $\begin{array}{l}\text { 11. Eu não incentivaria o uso do AVA aluno em } \\
\text { seu curso? }\end{array}$ & $\mathrm{E}$ & $\mathrm{D}$ & $\mathrm{D}$ & D & D & $\mathrm{E}$ & $\mathrm{D}$ & $\mathrm{E}$ & $60 \% \mathrm{D}$ \\
\hline $\begin{array}{l}\text { 12. A navegação proposta está mais fácil que a } \\
\text { atual. }\end{array}$ & A & A & B & A & B & A & $\mathrm{B}$ & B & $50 \%$ A, 50\% B \\
\hline $\begin{array}{l}\text { 13. Considero viável o site fornecer uma } \\
\text { autoajuda logo em seu primeiro acesso ou } \\
\text { sempre que precisar. }\end{array}$ & A & B & A & A & B & A & $\mathrm{B}$ & B & $50 \%$ A, 50\% B \\
\hline $\begin{array}{l}\text { 14. O site não deveria fornecer acesso direto ao } \\
\text { sistema de gestão acadêmica e ao sistema de } \\
\text { gestão do acervo da biblioteca da Universidade. }\end{array}$ & $\mathrm{E}$ & $\mathrm{E}$ & A & $\mathrm{D}$ & B & $\mathrm{E}$ & $\mathrm{D}$ & $\mathrm{D}$ & $\begin{array}{c}40 \% \mathrm{E}, 40 \% \mathrm{D}, 10 \% \mathrm{~A} \\
10 \% \mathrm{~B}\end{array}$ \\
\hline $\begin{array}{l}\text { 15. Considero que os recursos: aumentar fonte } \\
\text { da página, diminuir/aumentar contraste da } \\
\text { página, sejam importantes. }\end{array}$ & $A$ & B & A & B & B & $\mathrm{C}$ & $\mathrm{C}$ & C & $40 \%$ B, $40 \%$ C, $20 \%$ A \\
\hline
\end{tabular}

A partir da Tabela 3 é possível visualizar que 09 itens $(1,2,4,6,9,10,12,13,15)$ do questionário são de cunho positivo, e 05 itens $(3,5,7,8,11,14)$ são de cunho negativo; assim, os participantes precisariam estar atentos ao questionamento para poder marcar a alternativa adequada ao seu posicionamento.

Com isso, o resultado final da avaliação foi positivo. Analisando os dados da Tabela 3, de 120 possibilidades de respostas (8 usuários * 15 questões): 29 foram avaliadas com alternativa $A$ (Concordo fortemente); 43 com alternativa B (Concordo); $13 \mathrm{com}$ alternativa C (Nem concordo e nem discordo); $23 \mathrm{com}$ alternativa D (Discordo); e $12 \mathrm{com}$ alternativa $E$ (Discordo fortemente). Total de 72 A e B; 13 C e; 35 D e E. De 9 questões de cunho positivo (interpretação direta), foi obtido 25 de alternativas $A, 40$ de alternativas $B, 6$ alternativas $C, 1$ alternativa $D$ e 0 de alternativa E; ou seja, de 72 possibilidades de resposta, 65 foram avaliadas com A e B, somando quase $100 \%$ de possibilidade. De 6 questões de cunho negativo (interpretação invertida), foi obtido 4 de alternativas $A ; 3$ de alternativas $B ; 7$ de alternativas $C ; 22$ de alternativas $\mathrm{D}$; e 12 de alternativa $\mathrm{E}$; ou seja, de 48 possibilidades de respostas às questões de interpretação invertida, houve um total de 32 alternativas $D$ e $E$, somando mais da metade das possibilidades (questão negativa com resposta negativa = resultado positivo de avaliação).

Toda essa análise permite revelar que o redesenho do AVA aluno foi satisfatório para 100\% dos usuários entrevistados. Mesmo colocando as questões de forma negativa, mais que 50\% dos usuários discordaram, favorecendo a positividade das questões. Em suma, de 120 possibilidades, 97 foram positivas, 13 neutras e apenas 10 negativas. 


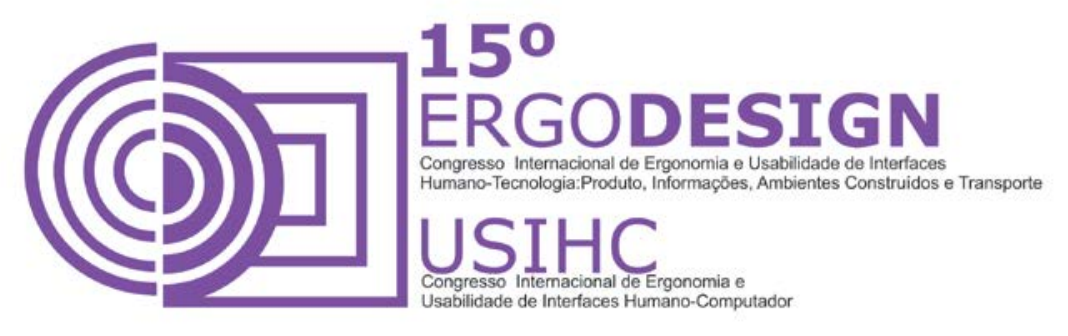

Todos os participantes voluntários elogiaram o novo redesenho do AVA. Vários participantes fizeram uma leitura parcial da tela antes de iniciar a tarefa proposta a eles. Alguns participantes clicaram em hiperlinks que a equipe de projeto não imaginaria que eles iriam buscar pela informação, deixando evidente que um novo ciclo de IHC seria necessário. Como comentário adicional (opcional), a participante E1 escreveu um depoimento em que informou que: "Gostaria que o AVA apresentasse grupos de interesse mesmo em cursos distintos, mas que apresentam disciplinas em comum". A funcionalidade de "grupos de interesse" seria apenas para os usuários do mesmo curso, a ideia da E1 foi considerada importante para aprimorar trabalhos futuros do redesenho do ambiente.

\section{CONCLUSÃO}

Como pode ser averiguado ao longo do artigo, nas primeiras etapas do projeto (análise contextual e concepção), não houve a participação de usuários. Devido a isto, houve muitas discussões em equipe sobre as ideias levantadas para o projeto de redesenho. Os projetistas também são alunos da Universidade em questão, o que auxiliou a elucidação de ideias em alguns momentos.

Foi satisfatório o uso da ErgoList, pois este checklist fornece um glossário das definições de cada critério, e permite que os projetistas efetuem a inspeção através de formulário online. No entanto, o laudo resultante do checklist somente disponibiliza os números (notas) da inspeção realizada para cada critério, os projetistas devem estar cientes disso antes de iniciar, assim não precisam realizar duplo trabalho posteriormente. À medida que se avalia o website, usando o checklist em tempo real, os projetistas necessitam ir efetuando as capturas de tela, com os recorte ou marcações, em paralelo para depois se lembrarem de que forma avaliaram cada elemento da interface.

Durante a avaliação do protótipo, ficou evidente que a participação do usuário, na primeira parte do projeto, seria de grande importância para elucidação de novas ideias. Este foi um aprendizado, por experiência prática, para a equipe de que a participação do usuário é bastante relevante para definir os requisitos de um sistema.

Como sugestão de continuidade de trabalhos similares, recomenda-se incluir no projeto questões associadas: à arquitetura da informação (CAMARGO e VIDOTTI, 2011) como a definição do sistema de navegação, de organização e de busca, à ampliação da interação social (ROGERS, SHARP e PREECE, 2013), e à acessibilidade (MELO e BARANAUSKAS (2005); WCAG 2.0 (2008); FERREIRA e NUNES (2008)).

\section{REFERÊNCIAS BIBLIOGRÁFICAS}

ANDRADE, R. S. S.; NEVES, P. T.. Utilização de um framework metodológico para avaliação da usabilidade do ambiente virtual de aprendizagem da Unimontes: Virtualmontes. Revista Multi[texto], Vol. 1, No. 1, 2012.

BARBOSA, J. G.; SÁ, S. R. de; GUSMÃO, E. H. O. de; VALENTIM, R. A. de M.; RÊGO, A. H. G. Usabilidade e navegabilidade no uso do Moodle: estudo de caso no Curso de Capacitação de 


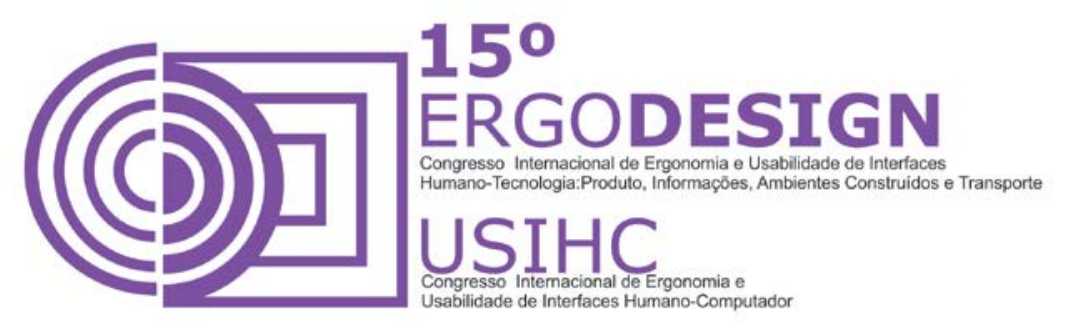

Gestores em Saúde do Ministério da saúde. Anais dos Workshops do CBIE, 2012.

CAMARGO, Liriane S. de A. de; VIDOTTI, Silvana A. B. G. Arquitetura da Informação: Uma Abordagem Prática para o Tratamento de Conteúdo e Interface em Ambientes Informacionais Digitais. Rio de Janeiro: LTC, 2011.

CARVALHO, Eduardo Costa de; ELIASQUEVICI, Marianne Kogut. Proposta de Melhoria na Interface do Moodle: Teste de Usabilidade com alunos do Curso de Bacharelado em Administração Pública na Modalidade à Distância da UFPA. X ESUD, Belém/PA, 2013.

CARVALHO, Rosângela S.; GOMES, A. S.; PERRIS, P. A. da R.; MELO Filho, I. J. de; FELICIANO, F. D. de O.; RODRIGUES, R. L. Análise comparativa da usabilidade dos ambientes de gestão da aprendizagem Amadeus e Moodle. RBIE, Volume 22, Número 1, 2014.

CYBIS, Walter, BETIOL, Adriana Holtz; FAUST, Richard. Ergonomia e Usabilidade: conhecimentos, métodos e aplicações. São Paulo, Novatec, 2007.

FERREIRA, Simone B. L.; NUNES, Ricardo R. e-Usabilidade. Rio de Janeiro: LTC, 2008.

GREENBERG, Saul; CARPENDALE, Sheelagh; MARQUARDT, Nicolai; BUXTON, Bill. Sketching User Experiences: The Workbook. Morgan-Kaufmann, 2012.

KRUG, Steve. Não me faça pensar: uma abordagem de bom senso à Usabilidade na Web. Alta Books: Rio de Janeiro, 2. ed., 2008.

MAGALHÃES, E.; Gomes, V.; RODRIGUES, A.; SANTOS, L.; CONTE, T. Impacto da usabilidade na educação a distância: um estudo de caso no Moodle IFAM. IX Symposium on Human Factors in Computing Systems (IHC '10), pp. 231-236, 2010.

MELO, A.M.; BARANAUSKAS, M.C.C. Design e Avaliação de Tecnologia Web-acessível. In: Anais do XXV Congresso da Sociedade Brasileira de Computação, Jornadas de Atualização em Informática, São Leopoldo, RS, pp. 1500 - 1544, 2005.

ROCHA, D. da S.; PALAZON, R. M. T. Avaliação comparativa dos aspectos de usabilidade das ferramentas de educação a distância (EAD) online Moodle, Teleduc e Planeta Rooda. Fasci-Tech Periódico Eletrônico da FATEC-São Caetano do Sul, São Caetano do Sul, v.1, n. 6, Mar./Set. 2012.

ROGERS, Yvonne; PREECE, Jennifer; SHARP, Helen. Design de interação: além da interação homem-computador; Porto Alegre, RS: Bookman, 2013.

SILVA, Solange Cristina da; BOCK, Geisa Letícia Kempfer; BECHE, Rose Clér Estivalete; GOEDERT, Lidiane. Ambiente Virtual de Aprendizagem Moodle: Acessibilidade nos Processos de Aprendizagem na Educação a Distância/CEAD/UDESC. X Congresso Brasileiro de Ensino Superior a Distância (ESUD), Belém/PA, 2013.

SILVEIRA, Clóvis; BATISTA, Marcelo H. E. Análise de softwares leitores de telas no Ambiente Virtual de Aprendizagem Moodle: Um estudo através de requisitos de qualidade de software. Revista iTEC, Vol. II, No 2, Jul. 2011.

VENKATESH, V.; MORRIS, M.; DAVIS, G.; DAVIS, F. User Acceptance of Information Technology: Toward a Unified View. MIS Quarterly, Vol. 27 No. 3, pp. 425-478, 2003. 